\title{
TRATAMIENTO AMBULATORIO DEL ABORTO INCOMPLETO
}

\author{
Doctor Carlos E. Sarria Olcos *
}

Cuando adelantaba mi especialización en el Instituto Materno-Infantil de Bogotá, golpeó mi curiosidad científica la posibilidad de estudiar en nuestro medio un tratamiento sencillo, rápido e inocuo en los innumerables casos de aborto incompleto que diariamente atendíamos. Era ese tratamiento, un método aplicado por el doctor David Braungardt y colaboradores en el sentido de practicar la terapia del aborto incompleto con raspado uterino, previa anestesia local y sin hospitalización posterior de la paciente. En esa idea, adelanté personalmente la investigación seleccionando cincuenta casos de pacientes con aborto incompleto en los cuales practicamos dicho tratamiento, cuyos detalles y pormenores quedaron consignados en las respectivas Historias Clínicas del Instituto, y a ellos quiero referirme en esta comunicación.

\section{PLAN DE TRABAJO}

Ya ingresada la paciente al Hospital y hecho el diagnóstico de aborto incompleto, si llenaba las condiciones requeridas, se le administraba la premedicación para luego proceder a la anestesia y raspado uterino. En el post-operatorio inmediato, control estricto cada hora por espacio de cuatro, momento en el cual se hacía el primer control definitivo. En ese momento se decidía el destino de la paciente que podía ser salida del Hospital u observación por dos horas más para completar seis horas de post-operatorio inmediato, al cabo de los cuales se practicaba el segundo control definitivo que daba de alta a la paciente o la hospitalizaba. En el post-operatorio tardío se hicieron controles a los tres, seis y diez días. De cada uno de los pasos enumerados anteriormente se hablará en detalle en rel desarrollo del estudio.

\section{CRITERIO DE ESCOGENCIA}

Hecho el diagnóstico de aborto incompleto se procedía a un detallado estudio de cada caso para concluir si

* Hospital San Juan de Dios. Bogotá, 1965. 
éste llenaba o no las condiciones requeridas para este trabajo. Dichas condiciones fueron las siguientes:

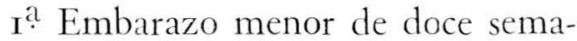
nas.

2. Estado del cuello uterino: dilatado o fácilmente "dilatable".

3. Aceptable estado general, con signos vitales dentro de límites normales.

$4^{\text {a }}$ Temperatura menor de $37 \cdot 5^{\circ}$.

Si el caso evaluado llenaba TODAS las condiciones enumeradas, fácilmente apreciables por el médico, se procedía a la pre-medicación anestésica para luego practicar el acto quirúrgico.

Como datos especiales se anotó en cada caso, y para efecto de estudios comparativos los siguientes: edad, paridad, edad del embarazo y abortos previos al actual.

Las cifras halladas fueron:

\section{Edad de la paciente}

\begin{tabular}{lrlr}
\hline Entre $15-20$ años & 5 casos & $10 \%$ \\
Entre 2I-25 años & I5 casos & $30 \%$ \\
Entre 26-30 años & I3 casos & $26 \%$ \\
Entre 3I-35 años & 7 casos & $14 \%$ \\
Entre 36-40 años & 8 casos & $16 \%$ \\
Entre 4 I-45 años & 2 casos & $4 \%$ \\
\hline
\end{tabular}

\section{Paridad}

La paridad de las pacientes intervenidas fue dividida en primigestantes, secundigestantes y multigestantes, correspondiendo $86 \%$ a estas últimas con un total de 43 casos; $10 \%$ a las secundigestantes con 5 casos y $4 \%$ a las primigestantes con dos casos:

\begin{tabular}{lrr}
\hline Primigestantes & 2 casos & $4 \%$ \\
Secundigestante & 5 casos & $10 \%$ \\
Multigestantes & 43 casos & $86 \%$ \\
\hline
\end{tabular}

\section{Edad del embarazo}

La edad del embarazo fue determinada únicamente por el tiempo de amenorea. El embarazo abortado tuvo marcada tendencia de la sexta a la cctava semana con un total de 3I casos que equivalen al $62 \%$ :

\begin{tabular}{|c|c|c|c|}
\hline & 4 semanas & & casos \\
\hline De & 5 semanas & I & caso \\
\hline De & 6 semanas & IO & casos \\
\hline De & 7 semanas & $I_{5}$ & casos \\
\hline De & 8 semanas & 6 & casos \\
\hline De & 9 semanas & 2 & casos \\
\hline De & Io semanas & 6 & casos \\
\hline De & I I semanas & 2 & casos \\
\hline De & I2 semanas & 3 & casos \\
\hline
\end{tabular}

Un dato importante que se anotó en cada historia fue el tiempo de evolución de la hemorragia vaginal. El cuadro siguiente da idea de la falta de preocupación de nuestra paciente hospitalaria ante un síntoma que como la hemorragia vaginal debiera hacer acudir a la paciente de inmediato a un servicio asistencial. También orienta este cuadro para apreciar la gran resistencia de nuestras pacientes a los dos peligros importantes del aborto incompleto, la anemia y la infección subsiguiente, pues a pesar 
de tener en la mayoría de los casos larga evolución en su sintomatología, estas complicaciones no se presentaron. El tiempo de evolución de la hemorragia vaginal fue:

\begin{tabular}{lrr} 
Menos de I2 horas & I4 casos & $28 \%$ \\
Entre 1/2-I día & 4 casos & $8 \%$ \\
Entre I-3 días & I 8 casos & $36 \%$ \\
Entre 4-7 días & 9 casos & $18 \%$ \\
Entre 8-Io días & 5 casos & $10 \%$ \\
\hline
\end{tabular}

Como último dato general anotado en cada caso, se tuvo en cuenta si la paciente presentaba como antecedente abortos previos. Se encontró un $46 \%$ correspondiente a 23 casos, en los cuales el antecedente de aborto anterior estaba presente.

\section{PREMEDICACION}

Si la paciente admitida con diagnóstico de aborto incompleto reunía todas las condiciones requeridas ya enumeradas, se procedía a la premedicación anestésica que se empezaba a administrar aproximadamente noventa minutos antes de efectuar el raspado, Esta premedicación consistia en: $x^{\circ}$. Dextrosa al $5 \%$ en agua destilada $500 \mathrm{cc}$. con diez unidades de Pitocin. $2^{\circ}$ Dextrosa al $5 \%$ en agua destilada $500 \mathrm{cc}$. con 50-100 mgrs. de clorhidrato de Meperidina, o en cambio de la solución dextrosada, 50-75 mgrs. de clorhidrato de Mepedirina diluídos en I8 cc. de agua destilada, y aplicados por vía endo-venosa.

El criterio para el uso y dosis del clorhidrato de Meperidina disuelto en goteo de Dextrosa o diluído en agua destilada e inyectado directamente en la vena fue el estado anímico de la paciente y la discutible apreciación objetiva del umbral de dolor en cada caso.

La premedicación empleada en los 50 casos fue como sigue:

I ${ }^{\circ}$ Dextrosa al $5 \%$ en agua destilada. $500 \mathrm{cc}$. con ro unidades de Pitocín, administrada a las 50 pacientes.

2. Clorhidrato de Meperidina:

a) En solución dextrosada:

$\begin{array}{rrr}\text { I00 mgr. } & 40 \text { casos } & 80 \% \\ 75 \text { mgr. } & 5 \text { casos } & 10 \% \\ 50 \text { mgr. } & 3 \text { casos } & 6 \% \\ \text { En I } 8 \text { cc de agua des- } & \end{array}$

b) En i8 cc de agua destilada

IOO gmr. 2 casos $\quad 4 \%$

\section{ANESTESIA}

Una vez administrada y evaluada la premedicación se procedía a la aplicación de la anestesia. Como método anestésico se usó en todos los casos el bloqueo útero-sacro, infiltrando únicamente a nivel de los ligamentos útero-sacros y a través del fondo de Douglas, dos puntos situados a las 4 y 8 del cuadrante del reloj e inmediatamente por debajo del cuelo uterino. No se infiltró en horquilla vulvar ni tampoco en el cuello.

El anestésico usado y sus diluciones fueron los siguientes:

\begin{tabular}{lrrr}
\hline Procaína al $2 \%$ & 3 casos & $6 \%$ \\
Xiloxaína al $1 \%$ & 24 casos & $48 \%$ \\
Xilocaína al $1 / 2 \%$ & 23 casos & $46 \%$ \\
\hline
\end{tabular}


Se hizo prueba conjuntival de sensibilidad a los anestésicos escogidos. La Xilocaína fue empleada SIN epinefrina y la cantidad de anestésico empleado en todos los casos aproximadamente roo cc. No se observó ninguna diferencia de acción entre las concentraciones del $1 / 2$ y $1 \%$ empleadas, recomendándose por lo tanto el uso de concentraciones al $1 / 2 \%$ ya que conlleva menos riesgo.

La evaluación de la acción anestésica y la tolerancia a la intervención se dividió en excelente, buena, regular y mala teniendo como criterio para su calificación el siguiente:

Excelente: Ninguna molestia para el paciente.

Buena: Discreta sensación dolorosa en hipogastrio.

Regular: Franco dolor en hipogastrio pero soportable.

Malo: Dolor acentuado en hipogastrio. Malestar general.

El resultado de la anestesia fue en los 50 casos así:

\begin{tabular}{lrr} 
Excelente & 36 casos & $72 \%$ \\
Buena & r I casos & $22 \%$ \\
Regular & 2 casos & $4 \%$ \\
Malo & I caso & $2 \%$ \\
\hline
\end{tabular}

La cifra de $72 \%$ con calificación excelente es un valor representativo de la efectividad de este método anestésico, que justifica su mayor empleo. La paciente con calificación anestésica mala (Caso $\mathrm{N}^{0}$ I5) no tuvo aparentemente ninguna causa que influyera en la mala acción anestésica. Se em- pleó Xilocaína al $\mathbf{~ \% ~ y ~ l a ~ p r e m e d i - ~}$ cación fue la usual.

\section{RASPADO UTERINO}

Ya aplicada y probada la anestesia se procedía al raspado uterino que se efectuó con la técnica usual teniendo especial cuidado de no traccionar el cuello. La no tracción del cuello uterino tiene como inconveniente al predisponer a una perforación uterina pues el ángulo cervico-corporal no se corrige; sin embargo no se observó ninguna complicación de este tipo. En todos los casos intervenidos se usó la cureta cortante fenestrada sin usar nunca la roma. No se emplearon valvas.

Durante los 50 raspados efectuados, 46 de los cuales fueron practicados por el autor de este estudio, únicamente en uno de ellos, el caso No 8, se presentó una pequeña complicación consistente en hemorragia eudouterina inmediatamente después del raspado y pese a tener la cavidad limpia e intacta. Cedió a un goteo de Dextrosa con ro unidades de Pitocín. Durante el raspado se suministró oxígeno a todas las pacientes. Luego del raspado y antes de salir a recuperación se administró en todos los casos maleato de ergonovina 0.2 grs. intramuscular, penicilina benzatínica 633 I. M. y por vía oral 0.5 grs. de piramidón.

Una vez hecho el raspado la paciente pasaba a recuperación, donde permanecía controlada de cerca, con estricta vigilancia y registro de tensión 
arterial, pulso y hemorragia vaginal. Estos datos se tomaron y anotaron cada hora por espacio de cuatro, para darle o no de alta. El criterio para ordenar la salida de la paciente se basaba en tres datos debidamente controlados y anotados a saber: tensión arterial, hemorragia vaginal y pulso.

a) TENSION ARTERIAL: Tomando las cifras de tensión arterial a la cuarta hora de post-operatorio inmediato se comparaba con la tensión registrada al ingreso y si aquella no era ostensiblemente menor $(20 \mathrm{~mm}$.) se consideraba que por esta causa se podía autorizar la salida de la paciente:

b) PULSO. La frecuencia y otras características del pulso fueron en las pacientes intervenidas, sensiblemente iguales al cabo de la cuarta hora que las obtenidas al ingreso. Hubo algunas variaciones en las primeras horas de control, pero al final de la cuarta hora (primer control definitivo) no se encontraron cambios significativos en las cifras anotadas.

c) HEMORRAGIA VAGINAL El tercer signo para decidir el destino de la paciente intervenida fue la hemorragia vaginal, dato que fue controlado cada hora al igual que los signos anteriores. De los 50 casos intervenidos, sólo uno, el número 6 , tuvo que ser demorado dos horas más, como medida preventiva, por haber presentado durante la primera hora del post-operatorio inmediato, hemorragia vaginal de mediana intensidad que cedió sin droga alguna, sólo con masaje uterino y expresión de algunos coágulos. En el resto de las pacientes la hemorragia vaginal fue la usual, luego de un raspado uterino.

De los cincuenta raspados efectuados, en cuarenta y nueve de ellos, los tres signos antes anotados fueron satisfactorios y permitieron apreciar la inocuidad del método. Sin embargo tres de ellas fueron dejadas en observación por dos hras más, luego del primer control definitivo (a la cuarta hora del post-operatorio inmediato) debido a lipotimias que se presentaron en el momento de levantarse la paciente. La causa puede atribuirse a hipotensión ortostática (casos 24-32 y 4I). Después de dos horas las enfermas se habían recuperado totalmente. Fueron cuatro las pacientes dejadas en observación, una por hemorragia y tres por lipotimias, pero todas ellas fueron dadas de alta en condiciones satisfactorias al cabo de 6 horas.

En resumen, de cincuenta pacientes intervenidas, cuarenta y seis salieron del servicio a la cuarta hora del postoperatorio y cuatro permanecieron por dos horas más.

\section{POST-OPERATORIO TARDIO}

Según el plan de trabajo previamente elaborado, practicamos controles a los tres, seis y diez días después de la intervención, y las pacientes acudieron cumplidamente como puede apreciarse en la siguiente relación:

Acudieron a los tres controles 35 pacientes, $70 \%$. 
Acudieron a dos controles I4 pacientes, $28 \%$.

Acudió a un solo control I paciente, $2 \%$.

Es de anotar que en los controles practicados no se evidenció ninguna complicación, cuando algunos autores han tenido con este mismo procedimiento infecciones pélvicas de todos los grados, llegando inclusive hasta la pelvi-peritonitis y peritonitis. También se han reportado casos de hemorragia vaginal por retención parcial de restos, dada la relativa dificultad de practicar el raspado uterino con anestesia local. En este estudio, únicamente una paciente (caso I) tuvo hemorragia vaginal tardía (el décimo día) que desapareció al tratamiento etinil-estradiol, en dosis de o,06 mgr. al día por veinte días. Hubo en otras pacientes relato de síntomas banales como cefalea, hipertermia y discretas hemorragias vaginales, sin que ninguno de estos síntomas se comprobara al control, ni tuvieran en revisiones posteriores alguna significación o secuela.

\section{VENTAJAS DE ESTE METODO}

Ventajas de tres órdenes, económicas, sociales y hospitalarias, tiene a mi entender este procedimiento.

a) Ventajas económicas. Son obvias para un medio hospitalario, donde el costo calculado cama-día es parte fundamental de su funcionamiento. Los cincuenta casos de este estudio fueron elaborados en un lapso de tres meses, recibiendo el autor aproximadamente un octavo del número de pacientes ingresadas al Instituto Materno Infantil. Si este método, luego de cuidadoso análisis y perfeccionamiento fuera adoptado por el Instituto, en tres meses se ahorrarían al presupuesto hospitalario, cuatrocientas hospitalizaciones cada una con permanencia rutinaria mínima de cuarenta y ocho horas, más el gasto de drogas correspondientes.

El factor económico es hoy en día de vibrante actualidad, dada la difícil situación financiera de los hospitales y la amenaza de suprimir asistencia hospitalaria por escasos recursos. Este método, sencillo y económico, podría adoptarse en nuestros hospitales y dada la gran cantidad de casos de aborto que llegan a ellos, contribuiría a solucionar en parte la falta de recursos económicos.

\section{Ventajas hospitalarias}

Desde el punto de vista movimiento hospitalario, y ya esbozado el factor económico, la agilización en la atención de paciente se aumenta considerablemente. Si a esto se añade la índole propia de un servicio obstétrico (urgencia permanente), lógicamente habrá más camas libres y más personal disponible para la atención de pacientes que sí requieren hospitalización y observación estrictas. Además a la paciente se le evita el riesgo anestésico que no deja de tener eventuales peligros.

\section{Ventajas sociales}

En nuestro medio y por las características propias de nuestra clientela 
hospitalaria, la cortísima permanencia en el Hospital, de cuatro a seis horas, evita el dejar abandonado el hogar muchas veces con los hijos, casi siempre numerosos, en condiciones infrahumanas, encerrados en una pieza y sin nadie que los atienda.
Hora de ingreso: II:45. Diagnóstico: Aborto incompleto de 8 semanas.

Raspado uterino: Hora: 13:15.

Anestesia Xilocaína $1 / 2 \%$ Io cc. Acción anestésica: Buena.

Post-operatorio inmediato: sale a recuperación con:

T. A. $12 \times 7$ Pulso $80 / \mathrm{m}$. No hay hemorragia vaginal Hora $14: 30$ T.A. $12 \times 7$ Pulso $80 / \mathrm{m}$. No hay hemorragia vaginal Hora $15: 30$ T.A. I2 $\times 8$ Pulso $80 / \mathrm{m}$. No hay hemorragia vaginal Hora $17: 30$. T.A. $12 \times 7$ Pulso $70 / \mathrm{m}$. No hay hemorragia vaginal Hora $16: 30$ T.A. $12 \times 7$ Pulso $70 / \mathrm{m}$. No hay hemorragia vaginal La paciente SALE del hospital.

HISTORIAS CLINICAS: A continuación se hace un resumen de las historias clínicas de las pacientes intervenidas. Unicamente se relatarán aquí las historias mencionadas en el transcurso del trabajo que merecen algún comentario especial. Como información general se transcribe el resumen de una historia tipo, similar a las de 43 pacientes que por su total normalidad no merecen comentario adicional.

HISTORIA TIPO: Caso No 46 I. M. 27 años. Hia. No I 160.095 - XII$6 / 63$.

G 8 P 5 A 2 Embarazo de 8 semanas.

Premedicación: Dextrosa 5\% A. D. 500 c. con ro p. Pitocín.

Dextrosa 5\% A. D. $500 \mathrm{cc}$. con 100 mgr. clorh. Meperidina.

T. A. $12 \frac{1}{2} \times 7$ - Pulso $75 / \mathrm{m}-\mathrm{T}^{\circ}$ 36.7.

Evolución de hemorragia: 4 días.
La paciente es dada de alta luego de cuatro horas de post-operatorio y acude luego a control 3,6 y io días después, es decir los días XII-9/63, XII-I2/63 y XII-I $8 / 63$ respectivamente, estando asintomática.

Las historias clínicas que por su evolución merecen algún comentario son reseñadas a continuación.

CASO No r. M. V. 26 años. Hia. No I52.250. Igreso VIII-30/63.

T. A. $12 \times 7-$ Pulso $80 / m-\mathrm{T}^{\circ}$ 36.7 .

$\mathrm{G}_{5} \mathrm{P}_{4}$ - Embarazo de 6 s:manas. Evolución de hemorragia: 2 días. Hora de ingreso: co:40. Diagnóstico: Aborto incompleto de 6 semanas.

Premedicación: Dextrosa 5\% A. D. 500 cc. con ro u. Pitocín.

Dextrosa 5\% A. D. $500 \mathrm{cc}$. con 100 mgrs. de Meperidina.

Raspado uterino: Hora: 2:00. Anestesia Xilocaína $1 \%$ Ioo cc. Acción anestésica: Exce'ente. 
Post-operatorio inmediato:

Hora $3: 10$ T.A. $9 \times 5$ Pulso $95 / \mathrm{m}$. No hay hemorragia vaginal Hora 4:10 T.A. $9 \times 5$ Pulso $95 / \mathrm{m}$. No hay hemorragia vaginal Hora 5:Io T.A. $9 \times 6$ Pulso $90 / \mathrm{m}$. No hay hemorragia vaginal Hora 6:10 T.A. i $x 7$ Pulso $85 / \mathrm{m}$. No hay hemorragia vaginal

La paciente SALE del hospital.

Post-operatorio tardío: La paciente acude a los controles a los 3,6 y io días, siendo éstos completamente normales. El $15^{\circ}$ días de post-operatorio, la paciente vuelve a consulta por hemorragia vaginal discreta, indolora y sin ningún otro síntoma agregado. El examen ginecológico es negativo Se formula etinil-estradiol $0.06 \mathrm{mgr} /$
Diagnóstico: Aborto incompleto de 4 semanas.

Premedicación: Dextrosa 5\% A. D. 500 cc. con ro u. Pitocín.

Dextrosa 5\% A. D. $500 \mathrm{cc}$.

Clorh. Meperidina ıoo gmr. en I8 cc. A. D. vía I. V.

Raspado uterino: Hora: 23:I0.

Anestesia Procaína 2\% Io cc.

Acción anestésica: Excelente.

\section{Post-operatorio inmediato:}

Hora co:ı T. A. I2 x 7 Pulso 8o/m. Hay hemorragia vaginal de mediana intensidad. Masaje uterino

Hora I:Io T.A. I2 $\times 7$ Pulso $80 / \mathrm{m}$. No hay hemorragia vaginal

Hora 2:I0 T.A. I2 $\times 8$ Pulso $80 / \mathrm{m}$. No hay hemorragia vaginal

Hora $3: 10$ T.A. I2 $\times 8$ Pulso $8 \mathrm{o} / \mathrm{m}$. No hay hemorragia vaginal

día durante 20 días. La hemorragia se suspendió al $2^{\circ}$ día de tratamiento y en un control posterior, 15 días después, estaba asintomática.

CASO No 6: E. R. 4I años. Hia. $\mathrm{N}^{0}$ 153.669. Ingreso: IX-9/63.

$\mathrm{G}_{5} \mathrm{P}_{4}$ Embarazo de 4 semanas.

T. A. $12 \times 7$ - Pulso $80 / \mathrm{m}$. - $\mathrm{T}^{\circ}$ $36.4^{\circ}$.

Hora de ingreso: 21:40.
En vista de que la paciente presentó durante la primera hora de postoperatorio hemorragia de mediana intensidad, se resuelve dejar la paciente en observación y como profilaxis por dos horas más. Al completar la sexta hora de post-operatorio, con signos, vitales normales y $\sin$ hemorragia vaginal, la paciente es dada de alta.

Los controles en el post-operatorio tardío fueron normales.

I. D. Hemorragia post-raspado por atonía uterina. 
CASO No 8. G. C. 35 años. Hia. $\mathrm{N}^{\circ}$ I5523I. Ingreso IX-25/63.

G 8 P 6 A I. Embarazo de 8 semanas.

T. A. $12 \times 7 \frac{1}{2}-$ Pulso $85 / \mathrm{m}-\mathrm{T}^{\circ}$ 36.8.

Hora de ingreso: 12:00

Diagnóstico: Aborto incompleto de 8 semanas.

Premedicación: Dextrosa 5\% A. D. 500 cc. con ro u. Pitocín.

Premedicación: Dextrosa 5\% A. D. $500 \mathrm{cc}$. con $100 \mathrm{mgr}$. de Clorh. Meperidina.

Raspado uterino: Hora: 13:30.

Anestesia Xilocaína I\% roo cc.

Acción anestésica: Buena.

Durante el raspado uterino hay hemorragia vaginal de mediana intensidad. La cavidad uterina se aprecia limpia e íntegra. Hay atonía uterina. Se ordena Dextrosa 5\% A. D. $500 \mathrm{cc}$. con ro u. Pitocín I. V., con lo cual cede la hemorragia en pocos minutos.
G 2 P I. Embarazo de 7 semanas.

T. A. $12 \times 7 \frac{1}{2}$ - Pulso $80 / \mathrm{m}-\mathrm{T}^{\circ}$ 36.7 .

Evolución de hemorragia: 3 horas. Hora de ingreso: 7:10.

Diagnóstico: Aborto incompleto de 7 semanas.

Premedicación: Dextrosa al 5\%. A. D. $500 \mathrm{cc}$. con io u. Pitocín.

Dextrosa 5\% A. D. $500 \mathrm{cc}$. con 100 mgr. clorh. Meperidina.

Raspado uterino: Hora: 8:40.

Anestesia Xilocaína $\mathrm{I} \%$ ro cc.

Acción anestésica: MALA. La paciente se queja de mucho dolor en hipogastrio. Malestar general. Náuseas. Hay evidentemente una mala acción anestésica que no tiene ninguna causa aparente ya que la técnica y droga empleadas así como la premedicación son las mismas usadas en el resto de pacientes.

Sin embargo el raspado se pued: hacer y los controles del post-operato-

\section{Post-operatorio inmediato:}

Hora i4:30 T. A. II $x 7$ Pulso $85 / \mathrm{m}$. Hemorragia vaginal muy discreta

Hora 15:30 T. A. I $x 7$ Pulso $85 / \mathrm{m}$. No hay hemorragia vaginal Hora $16: 30$ T.A. I $\times 7 \frac{1 / 2}{}$ Pulso $80 / \mathrm{m}$. No hay hemorragia vaginal Hora $17: 30$ T. A. I $\times 7 \frac{1}{2}$ Pulso $80 / \mathrm{m}$. No hay hemorragia vaginal

La paciente luego de la cuarta hora de post-operatorio sale del hospital Los controles del post-operatorio tardío 3, 6 y ro días después fueron normales. I. D. Hemorragia en el raspado por atonía uterina.

CASO No i5. B. V. 26 años. Hia. No 155.93 I. Ingreso: $\mathrm{X}-3 / 63$. rio inmediato son normales. La paciente sale del hospital a las cuatro horas y en los controles posteriores no se evidencia ninguna complicación I. D. Acción anestésica mala sin causa apreciable.

Los casos 24, 32 y 4 I pueden evaluarse y resumirse en una scla histo- 
ria ya que son en un todo similares. Los tres casos al finalizar la cuarta hora del post-operatorio fueron dados de alta y en el momento de incorporarse de la cama sufrieron lipotimia, pese a que minutos antes los signos vitales eran normales y semejantes a los del ingreso. La causa de este fenómeno es atribuible a hipotensión ortostásica por la permanencia en decúbito ayudada con la administración de Clorhidato de Meperidina en la medicación pre-anestésica. Estas tres pacientes fueron dejadas en observación dos horas más saliendo del hospital a las $6^{\text {a }}$ hora de practicado el raspado. De este grupo de pacientes se puede tomar como tipo la siguiente historia.

CASO No 32 T. R. 22 años Hia. No 158.330 . Ingreso XI-I6/63.

$\mathrm{G} 3 \mathrm{P}$ 2. Embarazo de ro semanas. T. A. I x 6 - Pulso 68/m - $\mathrm{T}^{\circ}$ 32.2. Evolución de hemorragia: 3 días.

Hora de ingreso: 12:30.

Diagnóstico: Aborto incompleto de io semanas.

Premedicación: Dextrosa 5\% A. D. $500 \mathrm{cc}$. con ro u. Pitocín.

Dextrosa 5\% A. D. 500 cc. $100 \mathrm{mgr}$. Clorh. Meperidina.

Raspado uterino: Hora: 14:00.

Anestesia Xilocaína 1/2\% ro cc.

Acción anestésica: Buena.
A esta paciente se le autoriza salida del hospital, mas al levantarse sufre lipotimia, con palidez marcada y suduración. Se examina la paciente y se hallan los signos de colapso vascular periférico. No se aprecia hemorragia vaginal. Se ordena cafeína I cc. I. M. y posición en decúbito supino. Al cabo de 30 minutos la paciente está restablecida y sale del hospital a la sexta hora de intervenida. Los controles del post-operatorio tardío son normales. Al igual que este caso número 32 , los casos 24 y 4 I tuvieron evolución semejante y el diagnóstico es igual para los tres. (Hipotensión ortostática post-raspado).

\section{CONCLUSIONES}

I.El tratamiento de aborto incompleto por el método empleado en este estudio, requiere una muy rigurosa selección de los casos pues la escogencia estricta y cuidadosa es el principal factor que determina el buen resultado final del mismo.

2. El método es de una rapidez que está de acuerdo con las exigencias actuales de agilización de nuestros servicios hospitalarios.

3. La premedicación debe emplearse con precauciones propias para cada caso y en las dosis ya señaladas. Se considera la buena premedicación co-

\section{Fost-operatorio inmediato:}

Hora i5:00 T. A. II x 6 Pulso $70 / \mathrm{m}$. No hay hemorragia vaginal

Hora $16: 00$ T. A. I $x 6$ Pulso $70 / \mathrm{m}$. No hay hemorragia vaginal

Hora i7:00 T. A. i $\times 7$ Pulso $70 / \mathrm{m}$. No hay hemorragia vaginal

Hora $18: 00$ T. A. i $x 7$ Pulso $70 / \mathrm{m}$. No hay hemorragia vaginal 
mo parte indispensable y fundamental de la buena acción anestésica.

4. El método de anestesia local (bloqueo útero-sacro) es muy eficaz y sus resultados justifican plenamente su empleo.

5. La técnica del raspado fue la usual, no habiendo tenido dificultades especiales.

6. Parte muy importante del éxito del método consiste en llevar controles rigurosos durante el post-operatorio inmediato y la directa vigilancia que se ejerza hora por hora sobre las pacientes.

$7^{\text {a }}$ Las drogas empleadas fueron mínimas y de muy bajo costo.

8a La estadía hospitalaria fue muy breve, siendo la máxima de seis horas

9. Los controles en el post-operatorio tardío fueron completos en la mayoría de los pacientes, hecho que no esperábamos dada la índole de las mismas. La mayor sorpresa de este estudio fue la total ausencia de complicaciones luego del raspado, pues autores extranjeros han tenido en estudios similares, complicaciones postoperatorias y aun en el mismo acto quirúrgico.

Io. Las ventajas económicas, sociales y hospitalarias justifican plena mente una cuidadosa evaluación del método y su posible adopción en nuestros congestionados servicios hospitalarios.

\section{BIBLIOGRAFIA}

1. BONICA: Tratamiento del dolor. Bloqueos regionales, 1961.

2. BRAUNGARDT DAVID: The out patient management of incomplete abortion. Amer. J. Obs. Gin. 6: 151, May. 15/63.

3. FREI J.: Tratado de anestesiología. Ooperaciones ginecológicas. Pág. 344, 1964.

4. SEEDS A.: Paracervical blocks. Obst. \& Gin. 20: 462, 1962.

5. SPANOS W. J.: Uterosacral block. Obs. \& Gin. 13: 129, 1959. 